\title{
Botulinum Toxin Treatment in the Healing of Pressure Ulcers Resulting from Spasticity in Multiple Sclerosis
}

Buchanan $\mathrm{K}^{1,2 *}$, Christofi $\mathrm{G}^{1,2}$, Farrell $\mathrm{R}^{1,2,3}$, Keenan $E^{1,2}$ and Stevenson $V^{1,2,3}$

${ }^{1}$ Department of Therapy and Rehabilitation, The National Hospital for Neurology and Neurosurgery, London, UK,

${ }^{2}$ UCLH NHS Foundation Trust, London, UK

${ }^{3}$ University College London, Institute of Neurology, London, UK

*Corresponding author: Buchanan K, Department of Therapy and Rehabilitation, The National Hospital for Neurology and Neurosurgery, PO Box 113, 2nd Floor, 8-11 Queen Square, London, WC1N 3BG, UK

Received: September 25, 2017; Accepted: October 17, 2017; Published: October 24, 2017

\begin{abstract}
Treatment of spasticity in patients with multiple sclerosis (MS) can be complex and challenging. Oral treatments can be poorly tolerated and despite their use, secondary complications such as contracture(s), bony deformity and pressure ulcers can ensue. We report the use of botulinum toxin (BoNT) injections to facilitate healing of pressure ulcers resulting from spasticity and spasm, in a patient with secondary progressive MS. The results suggest that treatment with BoNT, as part of a multi-disciplinary approach, may play a role in facilitating healing by reducing spasticity, spasm and pain, altering resting alignment and promoting more effective pressure relief.
\end{abstract}

Keywords: Spasticity; Spasm; Botulinum toxin; Pressure ulcers; Healing; Multiple sclerosis

\section{Introduction}

Botulinum toxin treatment offers a targeted approach to managing focal spasticity in a variety of neurological conditions $[1,2,3]$. In patients with lower limb spasticity, injection of BoNT has been shown to reduce hypertonia, increase passive range of motion, and reduce pain [4]. To date there are very few research studies evaluating the use of BoNT in the treatment of spasticity specifically in multiple sclerosis. Hyman et al [5] demonstrated a significant reduction in spasticity and increased ease of passive care when BoNT was administered to the hip adductors of patients with MS. The role of a combined approach of BoNT and physiotherapy in the treatment of MS related spasticity was evaluated by Giovanelli et al [6]. Results demonstrated a reduction in spasticity post BoNT and a suggestion that concomitant physiotherapy produces a better outcome.

A review of the literature supporting the use of BoNT as an adjunct to aid healing of pressure ulcers was undertaken. Two single case studies were identified $[7,8]$ involving patients who had developed spasticity, spasms or dyskinesia after sustaining either brain or spinal cord injury. Both patients developed pressure ulcers resistant to standard topical treatments. Targeted treatment with BoNT was administered into the muscles in proximity to the pressure ulcers and successful healing was achieved. The authors concluded that BoNT may facilitate breaking the cycle of stimulation of spasm, pain, poor resting posture and lack of pressure relief and its impact on healing of the ulcers.

\section{Case Presentation}

We present the case of a 51-year-old woman with secondary progressive multiple sclerosis (SPMS), experiencing spasms pulling her feet into plantarflexion and inversion, a progressive loss of range of movement at the ankles resulting in an abnormal resting position (Figure 1) and difficulty sourcing and applying appropriate footwear. In addition, she had developed significant painful, infected pressure ulcers of both feet. Other than Nabiximols (THC: CBD oromucosal spray: delta-9-tetrahydrocannabinol: cannabidiol: Sativex ${ }^{\infty}, \mathrm{GW}$

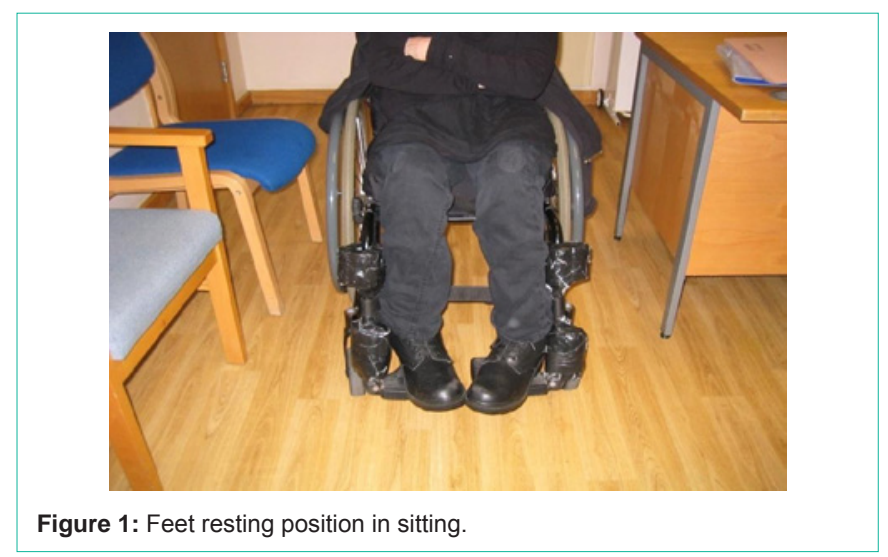

Figure 1: Feet resting position in sitting

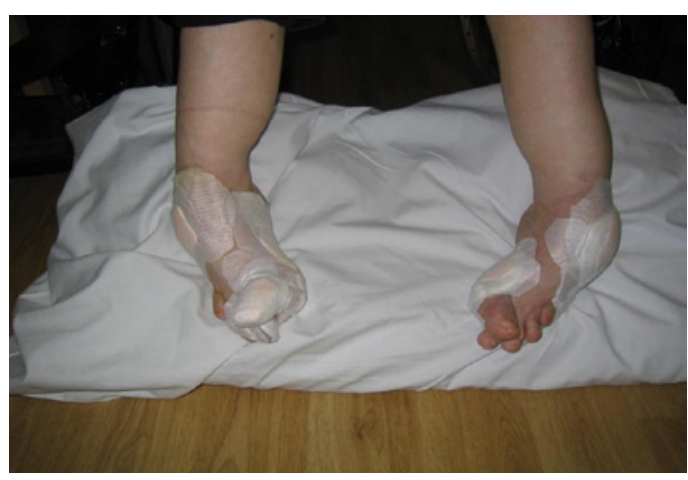

Figure 2: Pressure ulcer dressings on both feet.

Pharma Ltd, UK), which partially reduced her spasms, she was unable to tolerate oral antispasmodic medications due to side effects of sedation and weakness. Physical management strategies such as stretches and splinting had been trialled unsuccessfully due to the strength of the spasm and the poor skin quality. Intrathecal baclofen had been raised as a treatment option but the patient declined surgery. She had no other medical history; other medications included modafinil, calcium and vitamin D supplements.
Phys Med Rehabil Int - Volume 4 Issue 5 - 2017 ISSN : 2471-0377 | www.austinpublishing group.com Buchanan et al. ( ) All rights are reserved 
Table 1: Modified Penn Spasm Frequency Scale.

\begin{tabular}{|c|c|c|c|c|}
\hline \multirow[b]{2}{*}{ Side of body } & \multicolumn{2}{|c|}{ Pre-injection } & \multicolumn{2}{|c|}{4 weeks post-injection } \\
\hline & Left & Right & Left & Right \\
\hline Spasm frequency & 4 & 4 & 1 & 1 \\
\hline Spasm severity & 2 & 2 & 1 & 1 \\
\hline $\begin{array}{c}\text { Spasm description } \\
\text { (direction, duration, } \\
\text { spontaneous or triggered) } \\
\text { Plantarflexion: PF } \\
\text { Inversion: INV } \\
\text { Extension: ext }\end{array}$ & $\begin{array}{l}\text { Great toe ext, ankle PF } \\
\text { and INV, then knee ext }\end{array}$ & $\begin{array}{l}\text { Great toe ext, ankle PF } \\
\text { and INV, then knee ext }\end{array}$ & $\begin{array}{l}\text { Occasional mild spasms triggered by } \\
\text { end of range stretch; no toe involvement }\end{array}$ & $\begin{array}{l}\text { Occasional mild spasms triggered by } \\
\text { end of range stretch; no toe involvement }\end{array}$ \\
\hline
\end{tabular}

Table 2: Tone (Ashworth Scale) at initial assessment.

\begin{tabular}{|c|c|c|}
\hline Muscle group & Left leg & Right leg \\
\hline Hip flexors & 0 & 0 \\
\hline Hip extensors & 2 & 1 \\
\hline Knee flexors & 0 & 0 \\
\hline Knee extensors & 0 & 0 \\
\hline Ankle plantarflexors & 2 & 2 \\
\hline Ankle invertors & 2 & 2 \\
\hline
\end{tabular}

Despite once to twice weekly attendance to her local podiatry clinic to have the pressure ulcers dressed, they proved difficult to heal and she experienced two wound infections necessitating treatment with antibiotics. Osteomyelitis was ruled out.

Assessment of the lower limbs revealed 3 pressure ulcers in total: 2 on the left foot, one of which was oozing and had an offensive odour, and one on the right. In addition, there was an infected ingrowing great toe-nail (Figure 2 shows both feet with applications of dressings).

Any attempts at passive movement or stretch of either leg, elicited spasms with great toe extension and foot inversion. The Modified Penn Spasm Frequency Scale (MPSFS) [9], a self-report scale, used to assess a patient's perception of spasm frequency and severity was used as the primary outcome measure (Table 1). The MPSFS demonstrates excellent test-retest reliability when examined in the chronic spinal cord injury population [10]. Although its use has not been investigated in the MS population specifically, an assumption is made of the applicability to this patient group due to similarity in the clinical presentation. Resistance to passive movement was measured using the Ashworth Scale [11] and is detailed in Table 2. In view of the patient's poor alignment and loss of joint range of movement, resting position and passive range was also measured using goniometry, (Table 3) [12].

Despite maximising oral antispasmodic treatments, there were ongoing difficulties with healing of the pressure ulcers. Although this patient demonstrated a generalised picture of spasticity, in view of her intolerance to oral medication and following discussion with the patient, we proposed to target specific muscles with BoNT with goals of treatment being:

1. To reduce the number of spasms in her feet;

2. Improved resting alignment of her feet;

3. To facilitate better healing of her foot ulcers.

The patient was aware that the risks of treatment included muscle weakness and the potential impact of this on her ability to perform transfers.

BoNT type A (Allergan BOTOX ${ }^{\oplus}$ ) injections were undertaken under electromyography (EMG) guidance and muscle stimulation, to confirm localisation. Targeted muscles and treatment doses are detailed in Table 4. A second course of treatment to the same muscles was performed 4 months later. Post injection there was multidisciplinary team input from a number of specialties including: physiotherapy, podiatry, tissue viability and wheelchair services $[3,6]$.

\section{Results}

The outcome of the BoNT treatment for the patient was a reduction in spasms, a small improvement in foot resting position and passive range (Tables 1 and 3), increased ease of footwear application and ttolerance. All of her goals were achieved. The patient reported improved healing of the ulcers at 3 months and at 6 months with complete healing of the right foot ulcers and significant healing of the left foot ulcers. By approximately 9 months, the patient reported complete healing of all the ulcers.

\section{Discussion}

Spasticity and spasm can cause significant difficulties for many patients with MS, and if left untreated, can result in secondary complications such as contractures, joint deformities, pain and pressure ulcers. Within the National Health System (NHS) there is

Table 3: Goniometry of resting position and passive range of movement of the ankle.

\begin{tabular}{|c|c|c|c|c|c|}
\hline \multirow{2}{*}{ Limb Movement } & \multicolumn{2}{|c|}{ Resting Position } & \multirow[t]{2}{*}{ Limb Movement } & \multicolumn{2}{|c|}{ Passive Range of Movement } \\
\hline & $\begin{array}{c}\text { Pre } \\
\text { injection }\end{array}$ & $\begin{array}{c}4 \text { weeks Post } \\
\text { injection }\end{array}$ & & $\begin{array}{c}\text { Pre } \\
\text { injection }\end{array}$ & $\begin{array}{c}4 \text { weeks post } \\
\text { injection }\end{array}$ \\
\hline Left ankle plantarflexion & $55^{\circ}$ & $50^{\circ}$ & Left ankle dorsiflexion & $50^{\circ}$ off plantargrade & $45^{\circ}$ off plantargrade \\
\hline Left ankle inversion & $60^{\circ}$ & $40^{\circ}$ & Left ankle eversion & $30^{\circ}$ off neutral & $5^{\circ}$ off neutral \\
\hline Right ankle plantarflexion & $50^{\circ}$ & $40^{\circ}$ & Right ankle dorsiflexion & $50^{\circ}$ off plantargrade & $40^{\circ}$ off plantargrade \\
\hline Right ankle inversion & $50^{\circ}$ & $40^{\circ}$ & Right ankle eversion & $15^{\circ}$ off neutral & $10^{\circ}$ off neutral \\
\hline
\end{tabular}


Table 4: Targeted muscles and treatment doses.

\begin{tabular}{|c|c|c|}
\hline Left lower limb & BOTOX $®($ units) & Sites \\
\hline Tibialis posterior & 80 & 1 \\
\hline Tibialis anterior & 40 & 1 \\
\hline Hallucis longus & 40 & 1 \\
\hline Right lower limb & & 1 \\
\hline Tibialis posterior & 80 & 1 \\
\hline Tibialis anterior & 40 & 1 \\
\hline Hallucis longus & 40 & 6 \\
\hline Total dose & 320 & \\
\hline
\end{tabular}

a drive to reduce the incidence of pressure ulcers, not only because if left untreated they can prove fatal [13], but also due to the financial drain, with treatment costs ranging between $£ 43$-374 per day [14].

BoNT treatment aims to reduce focal spasticity and spasms and provides a window of opportunity to remediate associated problems such as adaptive soft tissue shortening, poor positioning and pain [4]. We postulate that it may play a role in facilitating the healing of pressure ulcers, as a secondary complication to spasticity, and this supposition is supported by the results of this case study. Larger studies are needed to further evidence these findings and inform future clinical practice. The local anti-inflammatory actions of botulinum toxin are not clearly established. In this case BoNT is unlikely to have played a significant role as the injections were administered proximal in relation to the pressure ulcers. BoNT treatment should be given as part of a coordinated MDT approach [3] and in this case, it is likely that the collaborative, patient-centered, MDT approach was a key component in achieving the outcome. An accurate assessment, firstly to identify the presence of spasticity and $\operatorname{spasm}(\mathrm{s})$, and secondly to define relevant treatment goals is essential. The use of additional patient reported outcome measures, such as the LegA [15] or the MSSS-88 [16] may be beneficial to assess the impact on ease of care and quality of life in future studies.

Our patient remains stable and continues to undergo twice yearly review including repeat BoNT treatment, with no signs of recurrence.

\section{Acknowledgements}

This project was supported by researchers supported by the National Institute for Health Research University College London Hospitals Biomedical Research Centre.

\section{References}

1. Stevenson VL, Jarrett L. Spasticity Management, a practical multidisciplinary guide, $2^{\text {nd }}$ Edition', New York: Taylor and Francis. 2016; 103.
2. National Institute for Health and Care Effectiveness. Guideline for the management of MS in primary and secondary care, London. 2014

3. Royal College of Physicians Spasticity management in adults: management using botulinum toxin. 2009

4. Olver J, Esquenazi A, Fung VSC, Singer BJ, Ward AB. Botulinum Toxin assessment, intervention and aftercare for lower limb disorders of movement and muscle tone in adults: international consensus statement. European Journal of Neurology. 2010; 17: 52-57.

5. Hyman N, Barnes M, Bhakta B, Cozens A, Bakheit M, Kreczy-Kleedorfer B, et al. Botulinum toxin (Dysport) treatment of hip adductor spasticity in multiple sclerosis: a prospective, randomised, double blind, placebo controlled, dose ranging study'. Journal of Neurology, Neurosurgery and Psychiatry. 2000; 68: 707-712.

6. Giovanelli M, Borriello G, Castri P,Prosperini L, Pozzilli C. Early physiotherapy after injection of botulinum toxin increases the beneficial effects on spasticity in patients with multiple sclerosis. Clinical Rehabilitation. 2007; 21: 331-337.

7. Intiso D, Basciani M. Botulinum Toxin Type A in the healing of a chronic buttock ulcer in a patient with spastic paraplegia after spinal cord injury'. J Rehabil Med. 2009; 41: 1100-1102.

8. Intiso D, Basciani M, Di Renzo F, Tolfa M, Grimalidi G, Fiore P. Botulinum Toxin Type $A$ in the healing of ulcer following oro-mandibular dyskinesia in a patient in vegetative state. J Rehabil Med. 2008; 40: 315-316.

9. Priebe MM, Sherwood AM. Clinical assessment of spasticity in spinal cord injury: A multidimensional problem. Arch Phys Med Rehabil. 1996; 77: 713716.

10. Adams MM, Ginis KAM, et al. The spinal cord injury spasticity evaluation tool: development and evaluation. Arch Phys Med Rehabil. 2007; 88: 1185-1192.

11. Ashworth B. Preliminary Trial of carisoprodal in multiple sclerosis. Practitioner. 1964; 192: 540-542.

12. Norkin CC, White DJ. Measurement of Joint Motion: A Guide to Goniometry. Philadelphia: PA: FA Davis. 1985

13. Brown, G. Long-term outcomes of full-thickness pressure ulcers: healing and mortality. Ostomy Wound Management. 2003; 49: 42-50.

14. Dealey C, Posnett J, Walker A. The cost of pressure ulcers in the United Kingdom. Journal of Wound Care. 2012; 6: 261-262, 264, 266.

15. Ashford S, Jackson D, Mahaffey P. Conceptualisation and development of the Leg Activity Measure (LegA) for patient and carer reported assessment of activity in the paretic leg. Physiotherapy Research International. 2016; 10: 1002/pri.1660.

16. Hobart JC, Riazi A, Thompson AJ. 'Getting the measure of spasticity in multiple sclerosis: The Multiple Sclerosis Spasticity Scale (MSSS-88). Brain. 2006; 129: 224-234.
Phys Med Rehabil Int - Volume 4 Issue 5 - 2017 ISSN : 2471-0377 | www.austinpublishing group.com Buchanan et al. @ All rights are reserved
Citation: Buchanan K, Christofi G, Farrell R, Keenan E and Stevenson VL. Botulinum Toxin Treatment in the Healing of Pressure Ulcers Resulting from Spasticity in Multiple Sclerosis. Phys Med Rehabil Int. 2017; 4(5): 1129. 